\title{
Supply Chain Network Optimization Based on Fuzzy Multiobjective Centralized Decision-Making Model
}

\author{
Xinyi Fu and Tinggui Chen \\ School of Management Engineering and Electronic Commerce, Zhejiang Gongshang University, Hangzhou 310018, China \\ Correspondence should be addressed to Tinggui Chen; ctgsimon@gmail.com
}

Received 17 October 2016; Accepted 19 January 2017; Published 5 March 2017

Academic Editor: Anna M. Gil-Lafuente

Copyright ( 2017 Xinyi Fu and Tinggui Chen. This is an open access article distributed under the Creative Commons Attribution License, which permits unrestricted use, distribution, and reproduction in any medium, provided the original work is properly cited.

\begin{abstract}
Supply chain cooperation strategy believes that the integration of the operation process can produce value for customers, optimize the supply chain of the connection between the vertical nodes, and constantly strengthen the performance of the advantages, so as to achieve mutual benefit and win-win results. Under fuzzy uncertain environment and centralized decision-making mode, we study multiobjective decision-making optimization, which focuses on equilibrium and compensation of multiobjective problems; that is to say, the proper adjustment of the individual goal satisfaction level will make other goals' satisfaction levels change greatly. Through coordination among the multiobjectives, supply chain system will achieve global optimum. Finally, a simulation experiment from Shaoxing textile case is used to verify its efficiency and effectiveness.
\end{abstract}

\section{Introduction}

If we take the supply chain network as a system to study, its involved scopes include cooperation relationship, logistics management, information flow management, enterprise's strategy formulation, tactical arrangement, and operation execution. The essence of effective supply chain network operation is a multiobjective optimization process under the constraints of internal and external factors [1]. These objectives include speed, flexibility, quality, cost, service, and other indicators according to actual situation. Because of different natures of supply chain networks, the target selection and the weight setting will be very different, which is closely related to the organization ways, decision structure, and decisionmaking model of supply chain networks [2]. Therefore, the multiobjective optimization of the supply chain network is also the decision-making optimization process.

Inventory management has always been a focus in supply chain management. Kacprzyk and Stanieski [3] and Park [4] were the first to introduce fuzzy mathematics into inventory management and extend the classical Economic Order Quantity (EOQ) model to the fuzzy inventory model under the condition of ordering cost and holding cost being fuzzy variables. Sadeghi et al. [5] developed a constrained VMI model with fuzzy demand for a single-vendor multiretailer supply chain, in which the centroid defuzzification method defuzzifies trapezoidal fuzzy numbers of the demand. Jana et al. [6] proposed some multi-item inventory models for deteriorating items in a random planning horizon under inflation and time value money with space and budget constraints. In addition, the models were illustrated with some numerical data and results for different achievement levels were obtained and sensitivity analysis on expected profit function was also presented. Mahata and Goswami [7] considered inventory models for items with imperfect quality and shortage backordering in fuzzy environments by employing two types of fuzzy numbers, which were trapezoidal and triangular, and two fuzzy models were developed. Finally, numerical examples were provided in order to ascertain the sensitiveness in the decision variables with respect to fuzziness in the components. De and Sana [8] dealt with a backorder EOQ model with promotional index for fuzzy decision variables and a graphical presentation of numerical illustrations and sensitivity analysis were done to justify the general model. Giannoccaro et al. [9] proposed a fuzzy echelon method to solve the inventory management in supply chain. Ouyang and Yao [10] set up a hybrid inventory model, where the annual demand quantity was fuzzy variables and 
lead time was a variable. Hsieh [11] studied the optimization of fuzzy production inventory model.

The application of fuzzy mathematics in the production management was first studied by Chanas et al. [12] who used it in the transportation problems. Besides, considering the premise of the customer demand quantity and external supplied quantity be discrete fuzzy variables, Petrovic et al. $[13,14]$ determined the order quantity and the lowest safety stock of the node enterprise so as to optimize the operation of the whole supply chain, through taking the satisfaction rate of cost and indent as the control target. Giannoccaro et al. [9] established the multistages inventory strategy to set up a reasonable inventory safety level in order to make the material of the whole supply chain hold the lowest cost, when market demand and inventory holding cost were fuzzy variables. Kannan et al. [15] presented an integrated approach, of fuzzy multiattribute utility theory and multiobjective programming, for rating and selecting the best green suppliers according to economic and environmental criteria and then allocating the optimum order quantities among them.

With the increasing integration of fuzzy theory and operations research, fuzzy programming is evolving from single-objective to multiobjective, from single layer to multilayer, and from single stage to multistage. Chen and Lee [16] study the multiobjective optimization problem of multilevel supply chain network under the condition of product demand and price being uncertain. Lin et al. [17] set up a fuzzy multiobjective integrated production planning model in the steel industry. Aliev et al. [18] developed a fuzzy multiobjective and multiproduct supply chain production and distribution model and used genetic algorithm to solve it. The experimental simulation demonstrated its efficiency and effectiveness. Rinks [19] used fuzzy logic method to develop the supply chain integrated production and planning model and problem-solving algorithm. Lee [20] established the fuzzy objective function of the single product integrated production plan in the different time stage. Gao et al. [21] used fuzzy reasoning Petri nets to establish a decision analysis system based on the electronic network supply chain. Finally, a comparative study on Pareto optimality and optimality under Lagrange's interpolating polynomial function had been made illustrating numerical example. Other similar literatures are as follows: interactive multicriteria fuzzy intensive production plan [22], double level optimization model for supply chain distribution system [23], production batch planning method with fuzzy capacity constraints [24], fuzzy multiobjective order allocation model, and so on [25].

This paper mainly studies multiobjective coordination of supply chain network under a fuzzy uncertainty environment and also explores the centralized decision-making method of multiobjective coordination of supply chain. The main contents in the paper focus on the optimization of mutual compensation in fuzzy multiobjective decision-making, so as to get the global optimization and determine the supply chain optimization subnet in this state.

The rest is organized as follows: in Section 2, centralized decision-making mode and fuzzy multiobjective analysis of supply chain are discussed. Section 3 proposes fuzzy multiobjective coordinated optimization method under the centralized decision-making method. Subsequently, the model is applied to a Shaoxing textile supply chain network and some discussions on the obtained results are also given in Section 4. Section 5 offers our concluding remarks and potential extensions of this research.

\section{Centralized Decision-Making Mode and Fuzzy Multiobjective Analysis of Supply Chain}

2.1. Centralized Decision-Making Mode of Supply Chain. Supply chain centralized decision mode refers to the advantage enterprises in the supply chain network with powerful strength, through the effective integration of information exchange system to integrate the other node enterprises in the supply chain network into a unified system for highly centralized decision-making and control management. Advantage enterprises eliminate antagonism between upstream and downstream enterprises, enhance the ability to resist risks, and achieve the mutual benefits and win-win of partners, through strengthening effective integration management of raw material supply, product manufacturing, distribution, and the whole sale process control [26]. In the centralized decision-making mode of the supply chain, the coordination of the multiobjective tasks can be realized through the effective control or influence by the unified decision-making layer, so as to improve the core competitiveness of the supply chain.

Multiobjective optimization of supply chain network is mutual coordination of the related steps in the objective function or the node enterprises. The supply chain network construction and optimization studied in this paper belong to the initial stage of supply chain network formation, and multiobjective selection needs to consider the whole supply chain, because the more objectives the optimization problem has, the greater the difficulty is. As a result, a complex supply chain multiobjective problem has difficulty in obtaining global optimization and can only achieve to the practical feasible solution after collaboration of all parties under practical constraints. Three objectives of optimized investment, setting cost, and satisfaction of customer service are considered at the same time when the production costs and node enterprises are into the optimization sub to operate.

2.2. Relevant Parameters' Definition of Multiobjective Analysis in the Centralized Decision-Making Mode of Supply Chain. In order to simplify the supply chain multiobjective model, the following assumptions are made:

(1) Locations of all nodes and various facilities in the supply chain network and the location are known.

(2) All node enterprises in supply chain network are fully cooperative ones and share information with each other.

(3) Do not consider the delay and lead time in the process of transportation, production, and distribution. 


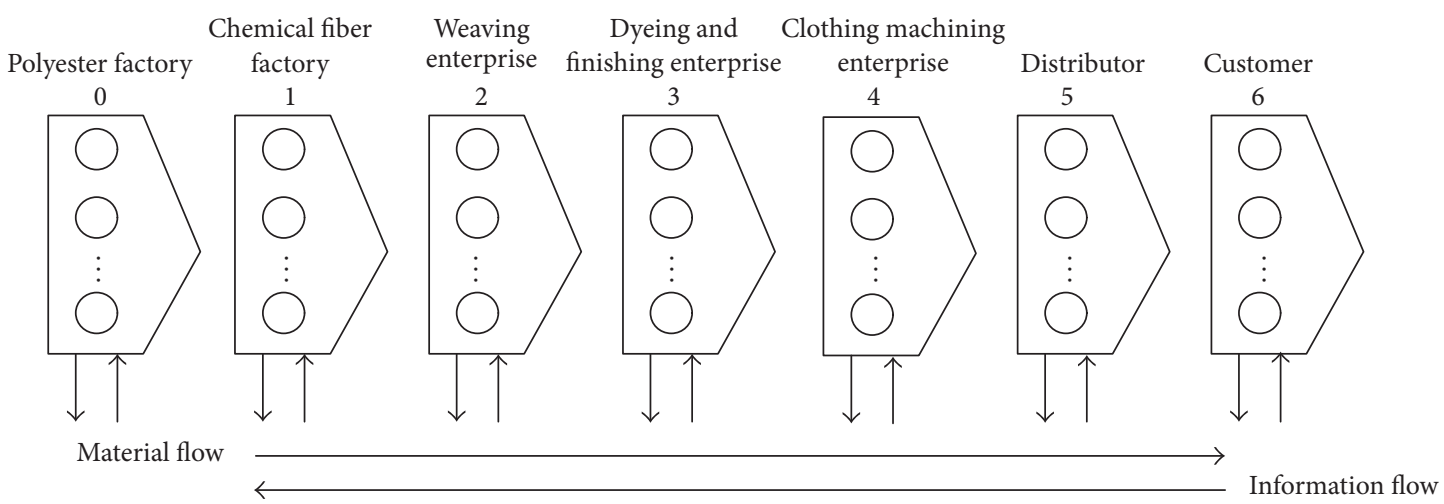

FIgURE 1: Hierarchical structure of textile supply chain.

(4) The performance or capacity of each node in the supply chain network is known.

In addition, related parameters used in the model are shown in Parameter Definitions.

\subsection{Fuzzy Multiobjective Mathematical Model of Supply} Chain. This paper takes the Shaoxing textile region network manufacturing as background. Shaoxing textile takes the industry of network organization as characteristics to get local industrial clusters, and the new model of regional economic development has been successfully deduced. At the same, it forms a complete industrial chain composed of the polyester, chemical fiber, weaving, dyeing, and finishing, clothing design and machining, sales, and so on. Hierarchical network structure of Shaoxing textile supply chain is shown in Figure 1.

The objective function of the supply chain network structure model is

$$
\min \tilde{f}_{1}=\sum_{i \in I} \sum_{j \in J} \widetilde{S}_{i j} X_{i j}+\sum_{j \in J} \sum_{k \in K} \widetilde{T}_{j k} Y_{j k}+\sum_{k \in K} \sum_{l \in L} \widetilde{U}_{k l} Z_{k l}+\sum_{j \in J} \widetilde{g}_{j} b_{j}+\sum_{k \in K} \widetilde{v}_{k} e_{k} .
$$

Under the supply chain network centralized decisionmaking, the unified decision-making layer always wishes to control the objective function $f_{1}$ to minimize the total cost. The total cost includes raw materials and transportation fuzzy costs of the weaving enterprises $\sum_{i \in I} \sum_{j \in J} \widetilde{S}_{i j} X_{i j}$, the transportation fuzzy costs $\sum_{j \in J} \sum_{k \in K} \widetilde{T}_{j k} Y_{j k}$ from production enterprises transferred to the clothing machining ones, the transportation fuzzy costs $\sum_{k \in K} \sum_{l \in L} \widetilde{U}_{k l} Z_{k l}$ from clothing processing enterprises to the distribution centers, the operation fuzzy costs $\sum_{j \in J} \widetilde{g}_{j} b_{j}$ producing the corresponding products in the dyeing and finishing enterprises in the unit time, and the corresponding operation fuzzy cost of clothing machining enterprises $\sum_{k \in K} \widetilde{v}_{k} e_{k}$.

$$
\min \tilde{f}_{2}=\sum_{j \in N} \widetilde{R}_{j} b_{j}+\sum_{k \in M} \widetilde{H}_{k} e_{k}
$$

In the process of supply chain network integration optimization, according to customer demand changes in different regions, decision-making layer conducts to start using the dyeing and finishing enterprises and clothing processing enterprises. In doing so, some input corresponding to these node enterprises will be produced in order to meet the demand of production or distribution of a particular product.
The objective function $f_{2}$ is defined as the minimum of the total running costs.

$$
\max \widetilde{f}_{3}=\frac{\sum_{k \in K} \sum_{l \in L} Z_{k i}}{\sum_{l \in L} \widetilde{d}_{l}}
$$

When the objective function $f_{3}$ gets a maximum, it indicates that decision-making layer hopes that the products of supply chain network maximization meet customer demands furthest.

The constraint conditions of the above objective functions are as follows:

$$
\begin{aligned}
& r \sum_{k \in K} Y_{j k} \leq \sum_{i \in I} X_{i j}, \\
& r \sum_{k \in K} Y_{j k} \leq p_{j} b,
\end{aligned}
$$

where formula (4) indicates that the raw material consumption in the production process of dyeing and finishing enterprises is restricted to the purchase quantity of raw materials in the weaving enterprises, and it is also affected by 
the actual production capacity constraints of the dyeing and finishing enterprises, which is shown in formula (5).

$$
\begin{aligned}
\sum_{j \in J} Y_{j k} & \leq \sum_{l \in L} Z_{k l}, \quad \forall k \in K, \\
\sum_{l \in L} \tilde{d}_{l} u_{k l} \leq q_{k} e_{k}, \quad \forall k \in K, & \\
Z_{k l} & \leq \tilde{d}_{l} u_{k l}, \quad \forall k \in K, \quad l \in L, \\
\sum_{j \in J} X_{i j} & \leq a_{i}, \quad \forall j \in J,
\end{aligned}
$$

where formula (6) expresses the constraint of the production quantity of the clothing enterprise; formula (7) indicates that the amount of demand determined by the distribution center should not be greater than the total capacity of the clothing machining enterprise; formula (8) indicates that the output of the goods in the clothing machining enterprise should not be greater than the total demand quantity of the distribution center, so as to avoid producing the inventory. Formula (9) is the raw material supply capacity constraints of the weaving enterprise.

$$
\begin{aligned}
\sum_{k \in K} u_{k l} & =1, \quad \forall l \in L, \\
\sum_{j \in J} b_{j} & \leq P, \\
\sum_{k \in K} e_{j} & \leq Q,
\end{aligned}
$$

where formula (10) ensures that each distribution center can obtain at least one garment processing enterprise service; formulas (11) and (12) are the number of restrictions of the available dyeing and finishing enterprises and clothing processing enterprises. Formula (13) is the two-dimensional variable constraint shown as follows:

$$
\begin{gathered}
b_{j} \in\{0,1\}, \\
e_{k} \in\{0,1\}, \\
u_{k l} \in\{0,1\},
\end{gathered}
$$

$$
\forall j \in J, k \in K, l \in L .
$$

Through discussion mentioned above, formulas (1) to (13) are composed of a fuzzy multiobjective model of supply chain.

$$
\begin{aligned}
\min & \tilde{f}_{1} \\
= & \sum_{i \in I} \sum_{j \in J} \widetilde{S}_{i j} X_{i j}+\sum_{j \in J} \sum_{k \in K} \widetilde{T}_{j k} Y_{j k}+\sum_{k \in K} \sum_{l \in L} \widetilde{U}_{k l} Z_{k l} \\
& +\sum_{j \in J} \widetilde{g}_{j} b_{j}+\sum_{k \in K} \widetilde{v}_{k} e_{k}
\end{aligned}
$$

$$
\begin{array}{ll}
\min & \tilde{f}_{2}=\sum_{j \in N} \widetilde{R}_{j} b_{j}+\sum_{k \in M} \widetilde{H}_{k} e_{k} \\
\max \quad & \tilde{f}_{3}=\frac{\sum_{k \in K} \sum_{l \in L} Z_{k i}}{\sum_{l \in L} \widetilde{d}_{l}} \\
\text { s.t. } \quad & r \sum_{k \in K} Y_{j k} \leq \sum_{i \in I} X_{i j}, \\
& r \sum_{k \in K} Y_{j k} \leq p_{j} b, \\
& \sum_{j \in J} Y_{j k} \leq \sum_{l \in L} Z_{k l}, \quad \forall k \in K, \\
& \sum_{l \in L} \widetilde{d}_{l} u_{k l} \leq q_{k} e_{k}, \quad \forall k \in K, \\
& Z_{k l} \leq \tilde{d}_{l} u_{k l}, \quad \forall k \in K, \quad l \in L, \\
& \sum_{j \in J} X_{i j} \leq a_{i}, \quad \forall j \in J, \\
& \sum_{k} u_{k l}=1, \quad \forall l \in L, \\
& e_{k \in K} \in\{0,1\}, \\
& \sum_{j \in J} b_{j} \leq P, \\
& \sum_{k \in K} e_{j} \leq Q,
\end{array}
$$

\section{Fuzzy Multiobjective Coordinated Optimization under the Centralized Decision-Making Method}

3.1. General Method of Fuzzy Multiobjective Coordination Optimization under the Centralized Decision-Making. In the process of multiobjective coordinated optimization, the objectives are conflicting and competing with each other. The optimization process cannot make all of objectives achieve the best, and the multiobjective problem is always to improve some objectives at the expense of others. In addition, the result of multiobjective optimization is based on the expectation of the decision-maker. However, it is difficult for decision-makers to express their goals or expectation precisely. Therefore, it belongs to fuzzy concept category. In this paper, the fuzzy theory is introduced to express the target satisfaction of the decision-makers by using the target membership degree and the feasible solution is also obtained through the coordination among the fuzzy satisfaction degree of each objective. 
The coordination-solving of fuzzy multiobjective problem can adopt the minimax operator, whose basic idea is that the decision-maker assigns a fuzzy expectation for each objective function in advance and uses the corresponding fuzzy set to represent it. The membership degree of fuzzy expectation represents the satisfaction of decision-maker with the corresponding objective and its solution is defined as the intersection of all fuzzy expectations. In doing so, a multiobjective problem can be transformed into an equivalent fuzzy single-objective one.

Let a multiobjective problem be

$$
\begin{array}{ll}
\max & \left\{f_{1}(x), f_{2}(x), \ldots, f_{k}(x)\right\} \\
\text { s.t. } & x \in X=\{x \in R \mid A x \leq b\},
\end{array}
$$

where $f_{k}(x)=c_{k}^{T} x(k=1,2, \ldots, K)$. Objective optimization process is as follow: set $x$ for $f_{k}(x) \geq f_{k}^{*}(k=1,2, \ldots, K, x \in$ $X)$, where $f_{k}^{*}$ is fuzzy expected value of corresponding objective function $f_{k}(x)$. If the objective of formula (15) is regarded as a fuzzy constraint and the allowable deviation of each fuzzy constraint is known, then the membership function $\mu_{k}(x)$ can be established. In the definition of "minimize" operators, the feasible solution set is defined as the intersection of all the target sets; that is to say,

$$
\begin{aligned}
\mu_{D}(x) & =\mu_{1}(x) \cap \mu_{2}(x) \cap \cdots \cap \mu_{K}(x) \\
& =\min \left\{\mu_{1}(x), \mu_{2}(x), \ldots, \mu_{K}(x)\right\} .
\end{aligned}
$$

If $\lambda=\mu_{D}(x)$, then formula (15) has the equivalent form shown as follows:

$$
\begin{array}{ll}
\max & \lambda \\
\text { s.t. } & \lambda \leq \mu_{k}(x), k=1,2, \ldots, K, \\
& x \in X .
\end{array}
$$

At this point, the decision-maker needs to determine the corresponding membership function of each target. For the $k$ th objective $(k=1,2, \ldots, K), f_{k}^{1}=\min _{x \in X} f_{k}(x)$ and $f_{k}^{0}=$ $\max _{x \in X} f_{k}(x)$ should be calculated firstly, and then according to the subjective preference the decision-maker chooses one of the functions from the linear function, the exponential function, the hyperbolic function, and the inverse hyperbolic function to construct a membership function. Generally, linear function is adopted. We can obtain

$$
\begin{aligned}
& \mu_{k}(x) \\
& = \begin{cases}1, & f_{k}(x) \geq f_{k}^{1}(x) \\
\frac{\left(f_{k}^{1}(x)-f_{k}(x)\right)}{f_{k}^{1}(x)}-f_{k}^{0}(x), & f_{k}^{1}(x) \geq f_{k}(x) \geq f_{k}^{0}(x) \\
0, & f_{k}(x) \leq f_{k}^{0}(x) .\end{cases}
\end{aligned}
$$

Note that the precondition of multiobjective problem shown in formula (15) or its equivalent form (shown in formula (17)) is the same weight of each target. In the practical problems of supply chain network, the importance of each target is different for the decision-maker. For example, in formulas (1) to (3), if we set different weights for these three objectives, then different optimal results will be obtained. At the moment, we introduce the objective weight of importance measures. Suppose the weight of the $k$ th $(k=1,2, \ldots, K)$ objective is $\omega_{k}$, and $\sum \omega_{k}=1$. In addition, set $\alpha=$ $\min \left\{\left(\mu_{1}(x)\right)^{\omega_{1}},\left(\mu_{2}(x)\right)^{\omega_{2}}, \ldots,\left(\mu_{K}(x)\right)^{\omega_{K}}\right\}$, and formula (17) can be further evolved into

$$
\begin{array}{ll}
\max & \alpha \\
\text { s.t. } & \alpha \leq \mu_{k}(x), k=1,2, \ldots, K, \\
& x \in X
\end{array}
$$

In the multiobjective decision-making, the weight is a number to describe relative importance of objectives, which often reflects the understanding of the decision-makers of decision-making problem. At the same time, the weight is also comprehensive reflection of the decision-makers' knowledge, experience, preferences, and so on. In centralized decision-making of supply chain, decision-makers should evaluate the influence degree of each objective for the supply chain operation, so as to reasonably determine the weight of each objective according to the actual situation. Because this paper only discusses the supply chain fuzzy multiobjective optimization to form the supply chain optimization subnetwork, the detailed process to determine the specific target weights is no longer expansion in the case simulation. In the centralized decision-making mode of supply chain, all the decision targets are included in the unified and centralized consideration of decision-makers and the weight sum of objectives at the same level is also equal to 1 ; that is to say, we suppose that the weight of the $k$ th $(k=1,2, \ldots, K)$ objective is $\omega_{k}$ and $\sum \omega_{k}=1$.

\subsection{Problem-Solving of Fuzzy Supply Chain Multiobjective} Optimization with Objective Satisfaction Compensation. The problem-solving of fuzzy multiobjective model mentioned above usually adopts the minimax operator, which only ensures that the satisfaction minimum goal in multiobjectives is to maximize satisfaction and others will be met by the minimum satisfaction. Therefore, the result is only a noninferior solution. Especially for solving complex multiobjective optimization problems which have multiple optimal solutions, this strategy cannot guarantee obtaining efficient solution. The reason is because the maximal and minimal operators are not compensatory ones. Subsequently, we integrate the minimal and compensatory arithmetic average operators and get the fuzzy minimal one which has certain compensation shown as follows:

$$
\mu_{f}(x)=\beta \min \mu_{D}(x)+(1-\beta) \frac{1}{K} \sum_{k=1}^{K} \mu_{k}(x) .
$$

Considering the impact of the objectives weight when the multiobjective is coordinated, formula (20) is further evolved, shown as follows:

$$
\mu_{f}(x)=\beta \min \mu_{D}(x)+(1-\beta) \sum_{k=1}^{K}\left(\mu_{k}(x)\right)^{\omega_{k}},
$$




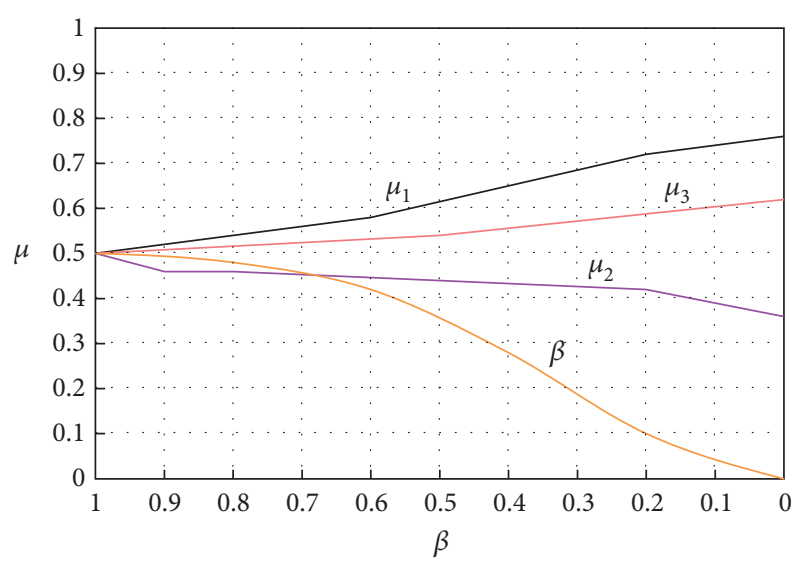

FIgURE 2: Coordination of fuzzy multiple objective satisfaction level based on compensation.

where $\beta$ is compensation coefficient and $0 \leq \beta \leq 1$. The main problem in using the compensation algorithm is how to find an appropriate parameter $\beta$ applicable to the decision function. As a result, we introduce the probability estimation of satisfactory range in advance for the involved objectives so as to achieve different satisfaction levels for different objectives. Figure 2 is the calculation results, which show that decision-makers can have better coordination by adjusting the value $\beta$ until the objectives achieve satisfaction so far.

3.3. Procedure of Supply Chain Fuzzy Multiobjective Optimization Solution. Using formula (21) and considering the importance weights of each objective, we propose optimization calculation formula based on compensation and interactive satisfaction for supply chain network multiobjective problem in formulas (1)-(13) shown as follows:

$$
\begin{array}{ll}
\max & \beta \lambda+(1-\beta) \sum_{k=1}^{K}\left(\mu_{k}(x)\right)^{\omega_{k}} \\
\text { s.t. } & \lambda \leq \mu_{k}(x), \quad k \in 1,2, \ldots, K, \\
& \lambda, \beta \in[0,1], \\
& \sum_{k=1}^{K} \omega_{k}=1 .
\end{array}
$$

The detailed procedures of optimization problem-solving are as follows.

(1) According to formulas (1)-(3), maximum $\left(f_{1}^{0}, f_{2}^{0}, f_{3}^{0}\right)$ and minimum $\left(f_{1}^{1}, f_{2}^{1}, f_{3}^{1}\right)$ values of objective function are obtained. Obviously, satisfaction solution of decision-makers for each objective function should be selected between the maximum and minimum values of each objective function under constraints.

(2) The membership of each objective function is constructed by formula (18), which represents the satisfaction degree of decision-makers to each objective in different values.

(3) Decision-makers determine the importance degree of each objective in the process of supply chain operation, so as to decide the weight of three objectives $\omega_{1}, \omega_{2}, \omega_{3}$, and $\omega_{1}+\omega_{2}+\omega_{3}=1$. At the same time, compensation coefficient $\gamma$ is also selected.

(4) Use formula (21) to obtain a compromise solution for the multiobjective problem. If the decision-maker accepts the compromise solution, then go to step (6) or continue to step (5).

(5) Compensation coefficient value $\gamma$ is revised by decision-maker. After that, go to step (4). At this time, the decision-maker also can adjust the objective function of maximal and minimal, and go to step (2).

(6) End up: the parameter value obtained by problemsolving standardizes operation relationship of supply chain network node enterprise, which determines the multiobjective coordination of the supply chain optimization subnetwork. The concrete flow is shown in Figure 3.

\section{Case Study}

Take Shaoxing textile supply chain network as the background; this network includes four levels $\{3-3-4-3\}$, that is, weaving enterprises $(I=3)$, dyeing and finishing enterprises $(J=3)$, clothing machining enterprises $(K=4)$, and distribution center $(L=4)$. Its network structure is shown in Figure 4. The advantage enterprises design supply chain and optimize operation process with the principle of lowest cost, lowest investment, and highest customer satisfaction. The detail parameter descriptions are shown in Table 1.

Under the constraint formulas (4)-(13), lingo 8.0 software is adopted to calculate the maximum and minimum value of the objective function in formulas (1)-(3). The final results are shown in Table 2.

The membership function of each fuzzy objective is obtained by formula (18):

$$
\begin{aligned}
& \mu_{1}(x) \\
& = \begin{cases}0, & f_{1}(x) \geq 4252 \\
4252-\frac{f_{1}(x)}{(4252-3586)}, & 4252 \geq f_{1}(x) \geq 3586 \\
1, & f_{1}(x) \leq 3586,\end{cases} \\
& \mu_{2}(x) \\
& = \begin{cases}0, & f_{2}(x) \geq 3360 \\
3360-\frac{f_{2}(x)}{(3360-2140)}, & 3360 \geq f_{2}(x) \geq 2140 \\
1, & f_{2}(x) \leq 2140,\end{cases} \\
& \mu_{3}(x)= \begin{cases}0, & f_{3}(x) \geq 1 \\
\frac{\left(f_{3}(x)-0.762\right)}{(1-0.762)}, & 1 \geq f_{3}(x) \geq 0.762 \\
1, & f_{3}(x) \leq 0.762 .\end{cases}
\end{aligned}
$$




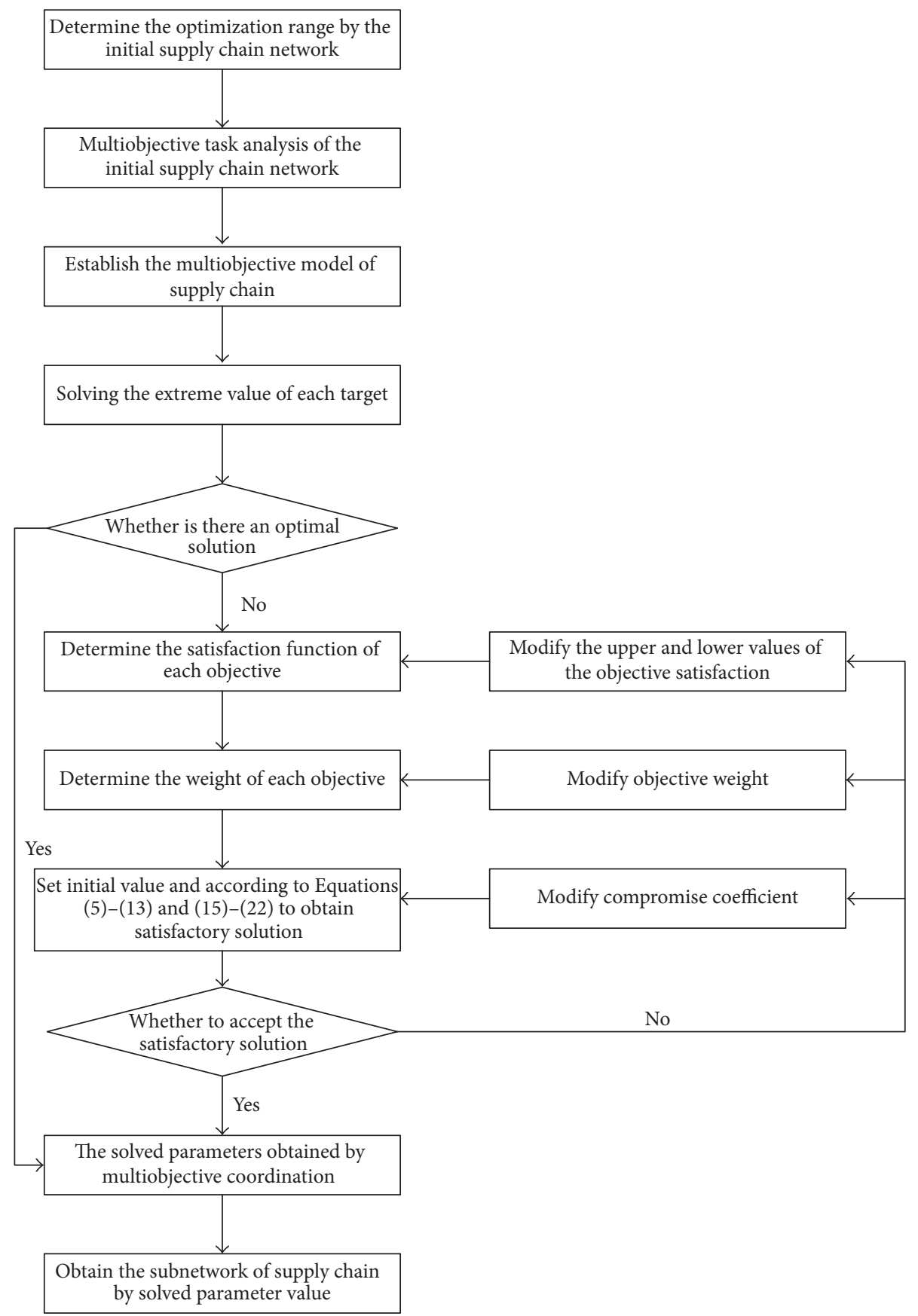

FIgURE 3: Fuzzy multiobjective optimization processes with compensation coefficient.

At first, the general interaction method is introduced to solve the optimization problem, and the membership functions of three objectives are substituted for formula (17). We can obtain the optimal satisfaction of decision-makers $\lambda^{*}=0.5103$, and the objectives are $f_{1}=3863, f_{2}=2737$, and $f_{3}=0.883$. If the decision-maker accepts the result, then end up; otherwise the maximum range and the membership function of each objective are adjusted to resolve until the decision-maker is satisfied. But, as previously mentioned, this method has not considered the relative importance of each target and satisfaction compensation in the objectives, so that the coordination effect of multiple objectives has some limitation.

Suppose the weights of three objectives are $\omega_{1}=0.4, \omega_{2}=$ 0.25 , and $\omega_{3}=0.35$. We can obtain different results through changing the value of $\gamma$ by calculating formula (21). It is shown in Table 3. The variation process of compensation coefficient can be expressed in Figure 2. Obviously, due to considering the weight and the compensation factor, the change rate of the satisfaction level in each objective is not the same with the change of $\gamma$. At this time, it is helpful for decisionmaker to search for optimization in the global scope. If 


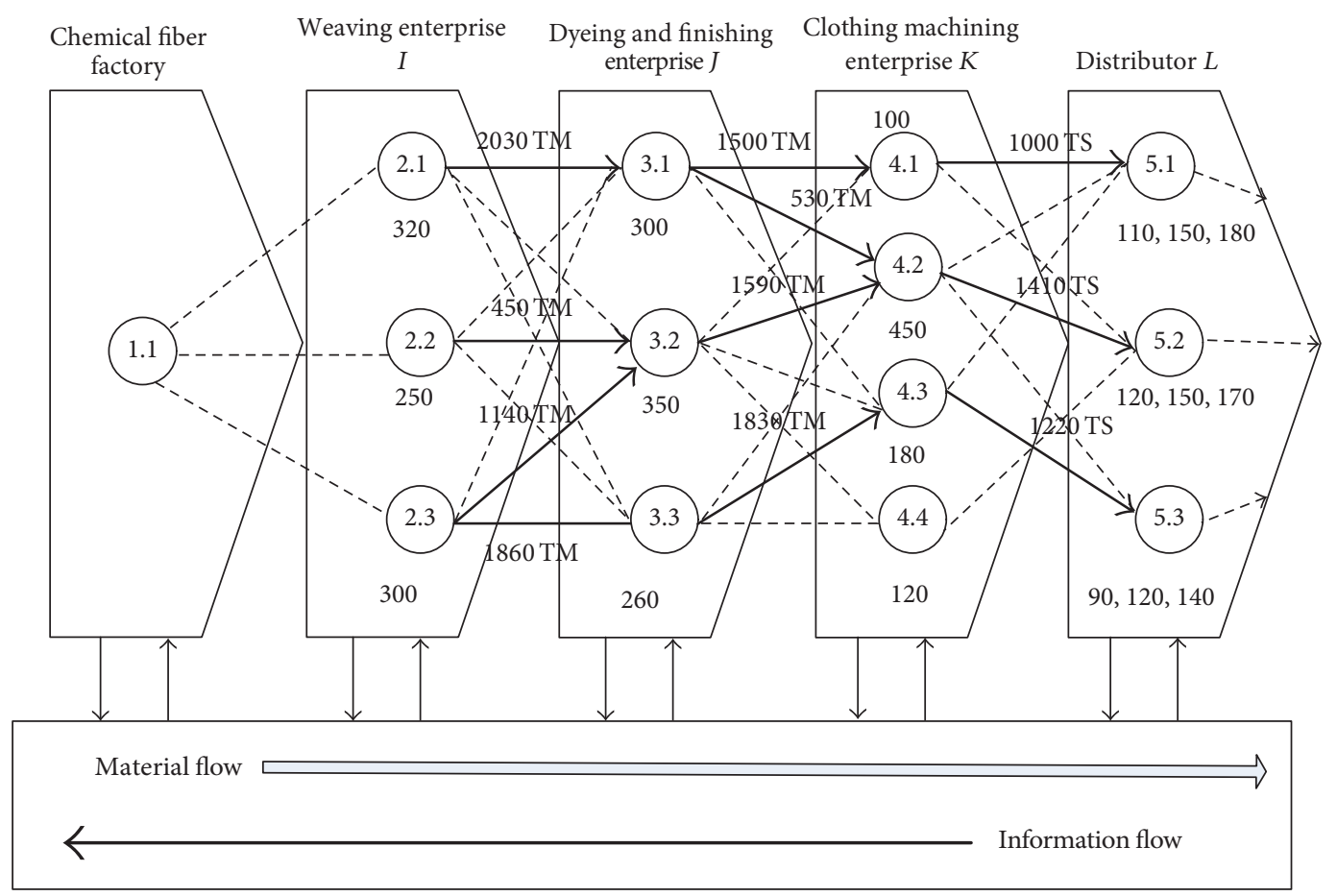

FIGURE 4: Supply chain network structure of Shaoxing textile. Note: TM means thousand meters and TS means thousand sets.

TABLE 1: Example of basic data table.

(a)

\begin{tabular}{lcccc}
\hline$\widetilde{s}_{i j}$ & $j=1$ & $j=2$ & $j=3$ & $a_{i}$ \\
\hline$i=1$ & $0.6,0.8,1.0$ & $0.4,0.6,0.8$ & $0.4,0.7,1.1$ & 320 \\
2 & $0.5,0.7,0.9$ & $0.6,0.8,1.0$ & $0.6,0.9,1.2$ & 250 \\
3 & $0.7,0.9,1.1$ & $0.3,0.7,0.9$ & $0.5,0.7,0.9$ & 300 \\
$\widetilde{p}_{j}$ & 300 & 350 & 260 & The material of each \\
$\widetilde{g}_{j}$ & $320,350,380$ & $300,320,340$ & $280,300,320$ & dress is 1.5 meters so \\
$\widetilde{R}_{j}$ & $650,700,750$ & $630,660,700$ & $620,650,680$ & $r=0.67$ \\
\hline
\end{tabular}

(b)

\begin{tabular}{lcccc}
\hline$\widetilde{T}_{j k}$ & $j=1$ & $j=2$ & $j=3$ & $\widetilde{v}_{k}$ \\
\hline$k=1$ & $1.0,1.2,1.4$ & $0.8,1.0,1.2$ & $1.2,1.5,1.8$ & $180,200,220$ \\
2 & $1.2,1.4,1.6$ & $1.2,1.4,1.6$ & $0.9,1.2,1.5$ & $220,240,260$ \\
3 & $0.9,1.2,1.5$ & $1.6,1.8,2.0$ & $0.8,1.0,1.2$ & $200,230,250$ \\
4 & $1.0,1.2,1.4$ & $1.1,1.4,1.7$ & $1.1,1.3,1.5$ & $230,250,270$ \\
\hline
\end{tabular}

(c)

\begin{tabular}{lcccc}
\hline$\widetilde{U}_{k l}$ & $l=1$ & $l=2$ & $l=3$ & $\widetilde{H}_{k}$ \\
\hline$k=1$ & $1.5,1.8,2.0$ & $1.6,1.8,2.0$ & $1.2,1.4,1.6$ & $250,270,300$ \\
2 & $1.2,1.4,1.6$ & $1.1,1.4,1.7$ & $1.1,1.3,1.5$ & $280,300,320$ \\
3 & $1.1,1.3,1.5$ & $1.5,1.7,1.9$ & $1.0,1.2,1.4$ & $340,360,380$ \\
4 & $1.0,1.2,1.4$ & $1.0,1.2,1.4$ & $0.9,1.1,1.3$ & $320,350,370$ \\
$\widetilde{d}_{l}$ & $110,150,180$ & $120,150,170$ & $90,120,140$ & \\
\hline
\end{tabular}


TABLE 2: The maximum and minimum values of multiple objectives.

\begin{tabular}{lcc}
\hline Objective & Maximum value $f_{k}^{0}$ & Minimum value $f_{k}^{1}$ \\
\hline Cost $f_{1}$ & 4252 & 3586 \\
Investment costs $f_{2}$ & 3360 & 2140 \\
Customer satisfaction $f_{3}$ & $1.00(100 \%)$ & $0.762(6.2 \%)$ \\
\hline
\end{tabular}

decision-maker considers that the solution of $\gamma=0.3 \mathrm{can}$ be accepted (that is to say, $f_{1}=3818, f_{2}=2826$, and $f_{3}=$ 0.902 ), then process-solving is over. The decision variables corresponding to multiobjective equations are shown in Table 4 .

\section{Conclusions}

This paper analyzes the centralized decision-making mode of supply chain network and illustrates the decision-making process and characteristics of the centralized decisionmaking. Based on the background of centralized decision, a fuzzy multiobjective model of supply chain has been established, which is based on the objective conditions and constraints of the initial optimization of the supply chain network. The multiple objectives of the supply chain network are all in the same optimization level in the centralized decision-making mode and the main difference lies in the important degree of each objective; that is to say, the objective's weight is different. Due to the limited resources of supply chain network, there are a lot of conflicts with multiobjective coordination. When exploring the general fuzzy multiobjective optimization methods, we focus on the equilibrium and compensation problem of multiobjectives, which means that proper adjustment of individual goal satisfaction level will make other goal satisfaction levels change greatly so as to achieve the better objective coordination. In the mathematics realization, decision-maker continuously changes the satisfaction level of each objective through the change of compensation coefficient in order to optimize and coordinate. Finally, the simulation results have testified that the model proposed in this paper is efficient and effective. Hence, we may conclude that this model is exclusively fitted for dealing with multiobjective decision-making optimization under fuzzy uncertain environment and centralized decision-making mode.

The potential extensions of this research may consider stochastic demand and imperfect supply chain systems in the proposed model. Other decision-making methods like group decision, multilevel decision, and so on may be included in this model in the future.

\section{Parameter Definitions}

$I, i$ : Weaving enterprise set, weaving enterprise identification

$J, j:$ Dyeing and finishing enterprise set, dyeing and finishing enterprise identification

$K, k$ : Clothing machining enterprises set, clothing machining enterprises identification
$L, l$ : Distribution center set, distribution center identification

$M$ : Actual available set of dyeing and finishing enterprises $(M \in J)$

$N$ : Actual available set of clothing machining enterprises $(N \in K)$

$\tilde{g}_{i}$ : Operation cost fuzzy number of dyeing and finishing enterprise $j$ in the unit time (ten thousand Yuan)

$\widetilde{v}_{k}$ : Operation cost fuzzy number of clothing machining enterprise $k$ in the unit time (ten thousand Yuan)

$\widetilde{S}_{i j}$ : Fuzzy number of unit product transportation cost from the weaving enterprise $i$ to the dyeing and finishing enterprises $j$ (Yuan)

$\widetilde{T}_{j k}$ : Fuzzy number of unit product transportation cost from the dyeing and finishing enterprise $j$ to the clothing machining enterprise $k$ (Yuan)

$\widetilde{U}_{k l}$ : Fuzzy number of unit product transportation cost from the clothing processing enterprise $k$ to the distribution center $l$ (Yuan)

$a_{i}$ : Raw material supply ability of weaving enterprise $i$

$p_{j}$ : Unit production capacity of dyeing and finishing enterprises $j$

$r$ : Production needed raw material consumption per unit product

$P: \quad$ Total number of dyeing and finishing enterprises that can be available or invested in construction

Q: Total number of clothing machining enterprises that can be available or invested in construction

$\widetilde{d}_{l}$ : The unit time quantity product demand fuzzy number of distribution center $l$ (ten thousand sets)

$\widetilde{R}_{j}:$ The operation cost fuzzy number of dyeing and finishing enterprises $j$

$\widetilde{H}_{k}$ : The operation cost fuzzy number of clothing machining enterprises $j$

$X_{i j}$ : Raw material transportation volume per time from the weaving enterprise $i$ to the dyeing and finishing enterprises $j$

$Y_{j k}$ : Product transportation volume per time from the dyeing and finishing enterprise $j$ to the clothing machining enterprises $k$

$Z_{k l}$ : Product transportation volume per time from the clothing machining enterprise $k$ to the distribution center $l$

$b_{j}$ : $0-1$ variable, where 1 indicates that the manufacturing plant is available; otherwise not

$e_{k}$ : $0-1$ variable, where 1 indicates that the clothing machining enterprise is available; otherwise not

$u_{k l}: 0-1$ variable, where 1 indicates the clothing machining enterprise service distributor $l$; otherwise not. 
TABLE 3: Corresponding compensation changes of the satisfaction degree of each objective.

\begin{tabular}{lccccccc}
\hline$\gamma$ & $\lambda$ & $f_{1}$ & $f_{2}$ & $f_{3}$ & $\mu_{1}$ & $\mu_{2}$ \\
\hline 1 & 0.5037 & 3917 & 2745 & 0.881 & 0.5037 & 0.5037 & 0.5037 \\
$0.9-0.8$ & 0.4875 & 3897 & 2780 & 0.884 & 0.5321 & 0.4752 \\
$0.7-0.6$ & 0.4291 & 3866 & 2795 & 0.886 & 0.5797 & 0.4635 \\
0.5 & 0.3625 & 3843 & 2809 & 0.888 & 0.6138 & 0.4517 \\
0.4 & 0.2936 & 3682 & 2807 & 0.897 & 0.6516 & 0.4531 \\
0.3 & 0.1886 & 3818 & 2826 & 0.902 & 0.6969 & 0.4375 \\
$0.2-0.1$ & 0.0628 & 3770 & 2869 & 0.905 & 0.7233 & 0.5311 \\
0 & 0.0000 & 3744 & 2914 & 0.910 & 0.7624 & 0.4022 & 0.3678 \\
\hline
\end{tabular}

TABLE 4: The parameters of the fuzzy multiobjective equation based on compensation.

(a)

\begin{tabular}{lcccc}
\hline$X_{i j}, b_{j}$ & $j=1$ & $j=2$ & $j=3$ & \\
\hline$i=1$ & $203 / 1$ & $/$ & $/$ & \\
2 & $/$ & $45 / 1$ & $/$ & \\
3 & $/$ & $114 / 1$ & $186 / 1$ & $Z_{k l}, u_{k l}$ \\
\hline
\end{tabular}

(b)

\begin{tabular}{lccccccc}
\hline$Y_{j k}, e_{k}$ & $j=1$ & $j=2$ & $j=3$ & $l=1$ & $l=2$ & $l=3$ & \\
\hline$k=1$ & $150 / 1$ & $53 / 1$ & $/$ & $100 / 1$ & $/$ & $/$ & $/$ \\
2 & $/$ & $159 / 1$ & $/$ & $/$ & $141 / 1$ & $/$ & $/$ \\
3 & $/$ & $/$ & $183 / 1$ & $/$ & $/$ & $122 / 1$ & $/$ \\
4 & $/$ & $/$ & $/$ & $/$ & & $/$ & $/$ \\
\hline
\end{tabular}

\section{Competing Interests}

The authors declare that there is no conflict of interests regarding the publication of this paper.

\section{Acknowledgments}

This research is supported by the National Natural Science Fund Project of China (Grant no. 71401156) and Higher Education Classroom Teaching Reform Project of Zhejiang Province in 2016 (Grant no. kg20160151) as well as Contemporary Business and Trade Research Center and Center for Collaborative Innovation Studies of Modern Business of Zhejiang Gongshang University of China (Grant no. 14SMXY05YB).

\section{References}

[1] T. Chen, C. Ju, and G. Zhou, "Disruption management for vehicle routing problem with time-window changes," International Journal of Shipping and Transport Logistics, vol. 9, no. 1, pp. 4-28, 2017.

[2] T. Chen and Y. Jiang, "Research on operating mechanism for creative products supply chain based on game theory," Discrete and Continuous Dynamical Systems. Series S, vol. 8, no. 6, pp. 1103-1112, 2015.

[3] J. Kacprzyk and P. Stanieski, "Long-term inventory policymaking through fuzzy decision-making models," Fuzzy Sets and Systems, vol. 8, no. 2, pp. 117-132, 1982.
[4] K. S. Park, "Fuzzy-set theoretic interpretation of economic order quantity," IEEE Transactions on Systems, Man and Cybernetics, vol. 17, no. 6, pp. 1082-1084, 1987.

[5] J. Sadeghi, S. M. Mousavi, and S. T. A. Niaki, "Optimizing an inventory model with fuzzy demand, backordering, and discount using a hybrid imperialist competitive algorithm," Applied Mathematical Modelling, vol. 40, no. 15-16, pp. 73187335, 2016.

[6] D. K. Jana, B. Das, and M. Maiti, "Multi-item partial backlogging inventory models over random planninghorizon in random fuzzy environment," Applied Soft Computing Journal, vol. 21, pp. 12-27, 2014.

[7] G. C. Mahata and A. Goswami, "Fuzzy inventory models for items with imperfect quality and shortage backordering under crisp and fuzzy decision variables," Computers and Industrial Engineering, vol. 64, no. 1, pp. 190-199, 2013.

[8] S. K. De and S. S. Sana, "Fuzzy order quantity inventory model with fuzzy shortage quantity and fuzzy promotional index," Economic Modelling, vol. 31, pp. 351-358, 2013.

[9] I. Giannoccaro, P. Pontrandolfo, and B. Scozzi, "A fuzzy echelon approach for inventory management in supply chains," European Journal of Operational Research, vol. 149, no. 1, pp. 185-196, 2003.

[10] L. Y. Ouyang and J. S. Yao, "A minimax distribution free procedure for mixed inventory model involving variable lead time with fuzzy demand," International Journal of Production Economics, vol. 76, no. 1, pp. 471-487, 2002.

[11] C. H. Hsieh, "Optimization of fuzzy production inventory models," Information Sciences, vol. 146, no. 1-4, pp. 29-40, 2002.

[12] S. Chanas, M. Delgado, J. L. Verdegay, and M. A. Vila, "Interval and fuzzy extensions of classical transportation problems," Transportation Planning and Technology, vol. 17, no. 2, pp. 203218, 1993.

[13] D. Petrovic, R. Roy, and R. Petrovic, "Supply chain modelling using fuzzy sets," International Journal of Production Economics, vol. 59, no. 1, pp. 443-453, 1999.

[14] D. Petrović, R. Petrović, and M. Vujošević, "Fuzzy models for the newsboy problem," International Journal of Production Economics, vol. 45, no. 1-3, pp. 435-441, 1996.

[15] D. Kannan, R. Khodaverdi, L. Olfat, A. Jafarian, and A. Diabat, "Integrated fuzzy multi criteria decision making method and multi-objective programming approach for supplier selection and order allocation in a green supply chain," Journal of Cleaner Production, vol. 47, pp. 355-367, 2013.

[16] C.-L. Chen and W.-C. Lee, "Multi-objective optimization of multi-echelon supply chain networks with uncertain product demands and prices," Computers and Chemical Engineering, vol. 28, no. 6-7, pp. 1131-1144, 2004.

[17] J. Lin, M. Liu, J. Hao, and S. Jiang, "A multi-objective optimization approach for integrated production planning under 
interval uncertainties in the steel industry," Computers and Operations Research, vol. 72, no. 8, pp. 189-203, 2016.

[18] R. A. Aliev, B. Fazlollahi, B. G. Guirimov, and R. R. Aliev, "Fuzzy-genetic approach to aggregate production-distribution planning in supply chain management," Information Sciences, vol. 177, no. 20, pp. 4241-4255, 2007.

[19] D. B. Rinks, "The performance of fuzzy algorithm models for aggregate planning and differing cost structures," in Approximate Reasoning in Decision Analysis, North Holland, Amsterdam, Netherlands, 1982.

[20] Y. Y. Lee, Fuzzy set theory approach to aggregate production planning and inventory control [Ph.D. thesis], Kansas State University, Manhattan, Kan, USA, 1990.

[21] M. Gao, M. C. Zhou, and Y. Tang, "Intelligent decision making in disassembly process based on fuzzy reasoning Petri nets," IEEE Transactions on Systems, Man, and Cybernetics Part B: Cybernetics, vol. 34, no. 5, pp. 2029-2034, 2004.

[22] Y. Dong, J. Tang, B. Xu, and D. Wang, "An interactive multi criteria fuzzy intensive production plan," Information and Control, vol. 33, no. 2, pp. 156-161, 2004.

[23] H. Sun and Z. Gao, "Bi-level optimization model of supply chain distribution system," Journal of Management Science Chain Distribution, vol. 6, no. 3, pp. 66-70, 2003.

[24] Y. Hong, X. Yang, and Y. He, "Research on the method of production lot planning with fuzzy capacity constraints," System Engineering Theory and Practice, vol. 21, no. 1, pp. 41-44, 2001.

[25] K. S. Moghaddam, "Fuzzy multi-objective model for supplier selection and order allocation in reverse logistics systems under supply and demand uncertainty," Expert Systems with Applications, vol. 42, no. 15-16, pp. 6237-6254, 2015.

[26] T. Chen and R. Xiao, "Modeling design iteration in product design and development and its solution by a novel artificial bee colony algorithm," Computational Intelligence and Neuroscience, vol. 2014, Article ID 240828, 13 pages, 2014. 


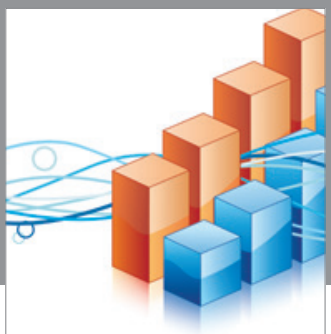

Advances in

Operations Research

vatem alat4

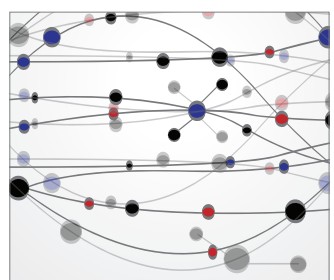

\section{The Scientific} World Journal
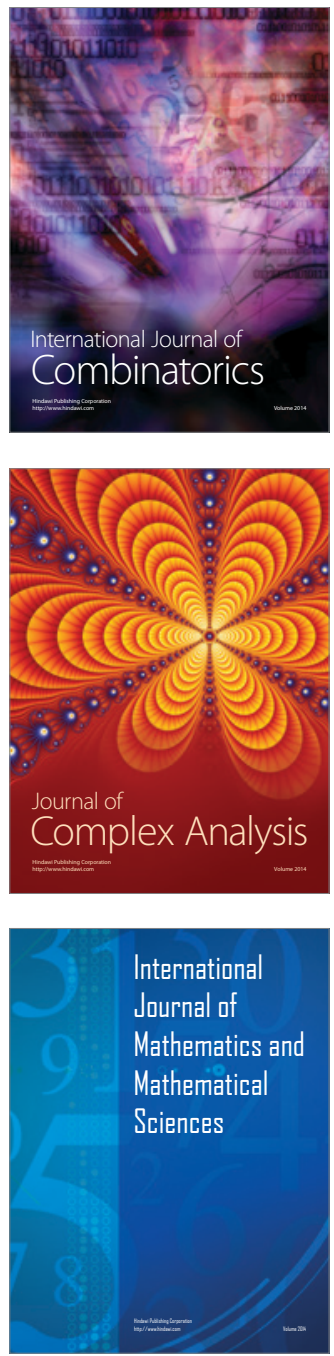
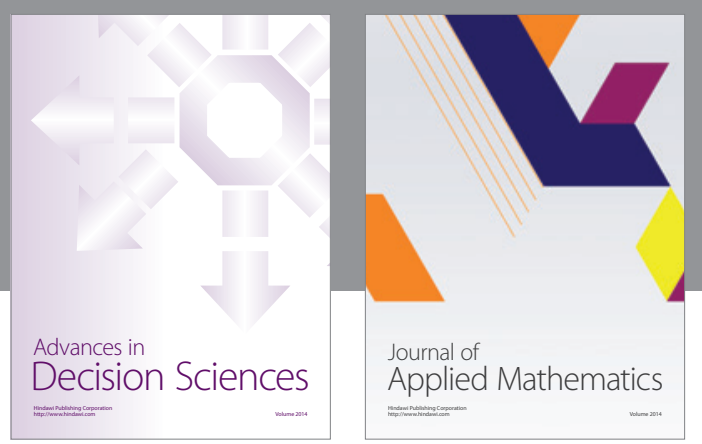

Algebra

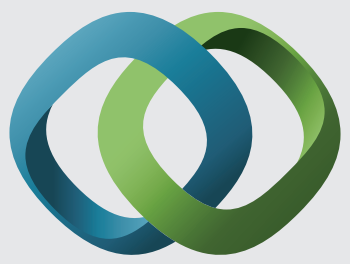

\section{Hindawi}

Submit your manuscripts at

https://www.hindawi.com
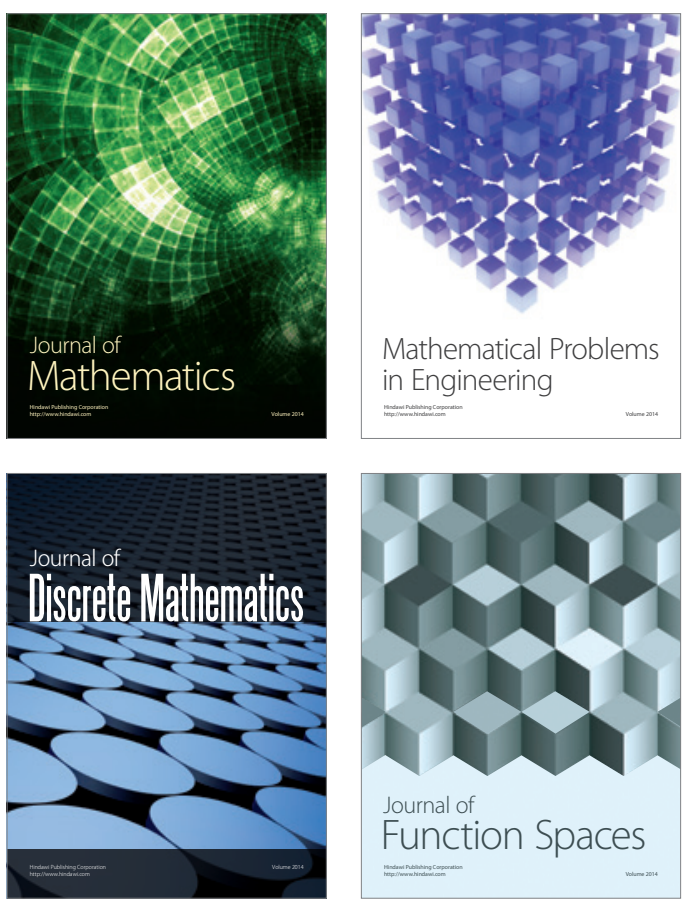

Mathematical Problems in Engineering
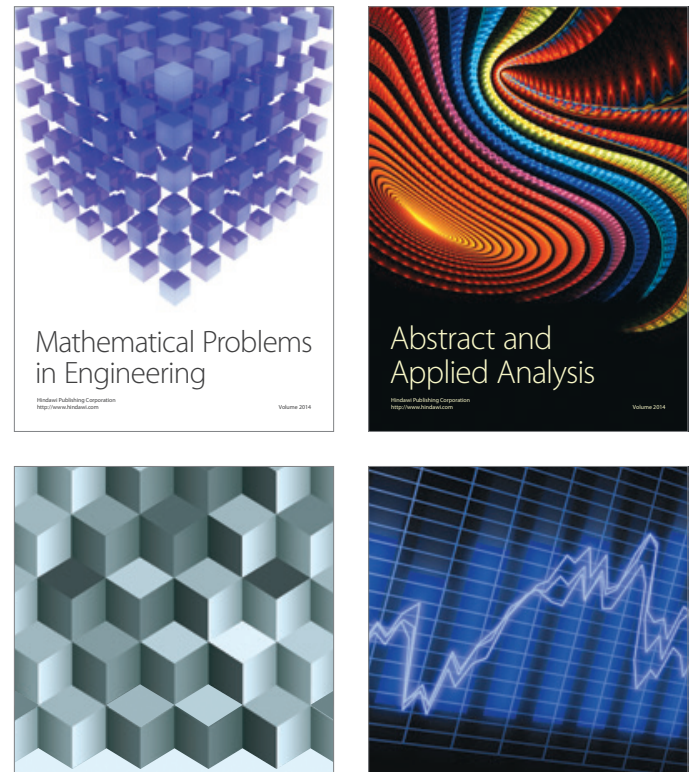

Journal of

Function Spaces

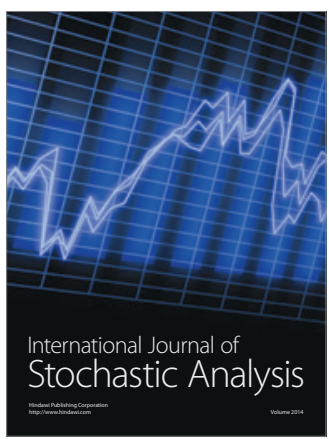

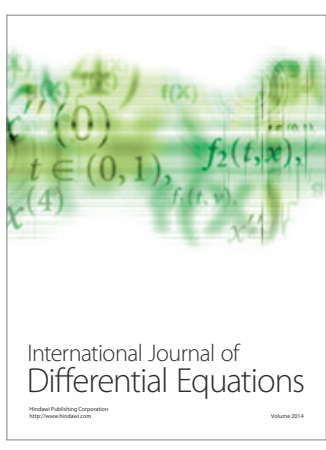
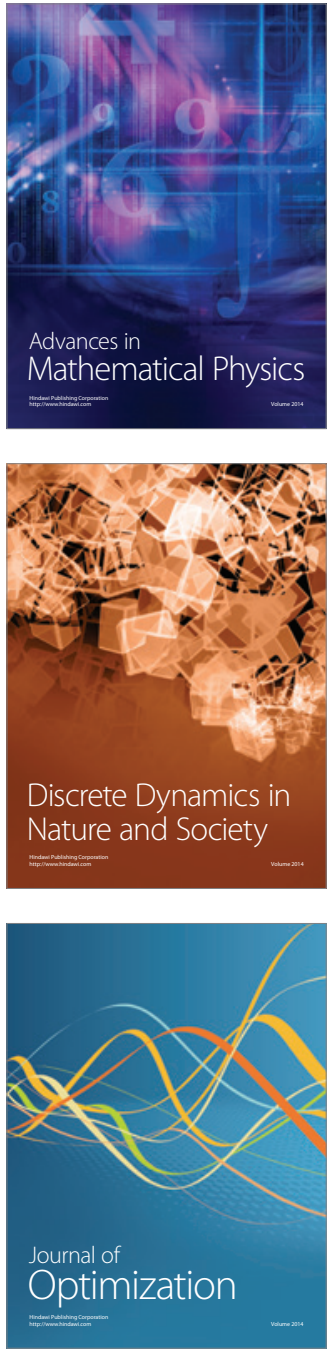